M. ITOH

KODAI MATH. J.

11 (1988), 344-360

\title{
GAUGE FIELDS AND QUATERNION STRUCTURE
}

\author{
By MitsuHiRo ITOH
}

Dedicated to Professor M. Obata on his 60th birthday

1. The aim of this article is to discuss geometry of the moduli space of Yang-Mills connections over a 4-manifold with quaternion structure.

Let $(M, h)$ be a compact, connected Riemannian 4-manifold with covariantly constant almost complex structures $\left\{I_{1}, I_{2}, I_{3}\right\}$ satisfying $I_{1} I_{2}=-I_{2} I_{1}=I_{3}$. We call such almost complex structures covariantly constant quaternion structure. Note that only complex flat two-tori and Ricci flat $K 3$ surfaces are such spaces.

Each almost complex structure $I_{\imath}$ given on the base space $M$ defines a 2 form $\theta_{2}$ on $M$ which is covariantly constant; $\theta_{i}(X, Y)=h\left(I_{2} X, Y\right), i=1,2,3$. The manifold $M$ carries the canonical orientation compatible with the quaternion structure. The base metric $h$ together with this orientation gives the Hodge operator $* ; \Lambda^{2}(M) \rightarrow \Lambda^{2}(M)$, which is involutive. So the bundle $\Lambda^{2}=\Lambda^{2}(M)$ splits into $\Lambda^{2}=\Lambda^{+}+\Lambda^{-}\left(\Lambda^{+}\right.$and $\Lambda^{-}$are subbundles of self-dual 2 -forms and of antiself-dual 2 -forms, respectively). Then over the manifold $M \Lambda^{+}$becomes trivial. We have indeed the decomposition;

$$
\Lambda^{+}=\boldsymbol{R} \theta_{1} \oplus \boldsymbol{R} \theta_{2} \oplus \boldsymbol{R} \theta_{3}
$$

Let $P$ be a smooth principal bundle over the manifold $M$ with a compact simple Lie group $G$.

Fix a positive number $l>4$ in order that analysis on gauge fields works well and denote by $A=A_{P}$ the set of all $L_{l}^{2}$ connections on $P$. The set $A$ is an affine space with model vector space $\Omega^{1}\left(\mathrm{~g}_{P}\right)_{l}$, the space of $L_{l}^{2} 1$-forms over $M$ taking values in the adjoint bundle $\mathrm{g}_{P}=P \times_{A d} \mathrm{~g}(\mathrm{~g}$ is the Lie algebra of $G)$. Then $\mathcal{A}=A+\Omega^{1}\left(\mathfrak{g}_{P}\right)_{l}$ for some fixed smooth connection $A$. The subset $\mathcal{A}_{\imath r}$ in $A$ consisting of irreducible connections is dense and open relative to the $L_{l^{-}}^{2}$ topology. A connection is said to be irreducible if the centralizer of its holonomy group in $G$ reduces to the center $Z_{G}$ of $G$.

The group $\mathcal{G}=\mathcal{G}_{P}$ of $L_{l+1}^{2}$ gauge transformations of $P$ acts on $\mathcal{A}$ smoothly as $g(A)=g^{-1} d g+g^{-1} \cdot A \cdot g$. Remark that $\mathcal{G} / z_{G}$ acts freely on $\mathcal{A}_{2 r}$ so that by the slice argument $\mathcal{A}_{\imath r}$ has a fibration over the orbit space $\mathcal{B}_{\imath r}=\mathcal{A}_{\imath r} /\left(g /_{Z_{G}}\right)$ with fibre $\mathcal{G} / z_{G}$.

Received January 18, 1988 
A connection $A$ is $\mathrm{ASD}$ (anti-self-dual) if and only if its curvature $F=F(A)$ $=d A+1 / 2[A \wedge A]$ sati-sfies the anti-self-duality equations

$$
F(A)+* F(A)=0 \text {. }
$$

Since $F(g(A))=g^{-1} \cdot F(A) \cdot g, g \in G / Z_{G}$, the solution space $\mathcal{A}^{-}$of $(1.2)$ is invariant under the gauge action. Hence we have the quotient $\mathscr{M}=\mathcal{A}^{-} /\left(\mathscr{L} / z_{G}\right)$ which parametrizes the set of gauge equivalence classes of solutions. The quotient space is called the moduli space of ASD connections on $P$.

Relative to geometrical structure of the moduli space over a general compact, oriented Riemannian 4-manifold $(M, h)$ we have the following finite dimensional space theorem.

THEOREM ([1]). The moduli space $\mathscr{M}$ of ASD connections on a principal bundle $P=P(M, G)$ is a smooth Hausdorff manifold possibly with singularities provided $\mathscr{M}$ is not empty. The dimension of the generic part $\operatorname{dim}_{R} \mathscr{M}$ is $p_{1}\left(g_{P} \otimes C\right)$ $[M]-\operatorname{dim}_{R} G\left(1-b_{1}+b^{+}\right)$, where $b_{1}$ is the first Bettr number of $M$ and $b^{+}=$ $\operatorname{dim}_{R} H^{+}(M), H^{+}(M)=\{$ self-dual harmonic 2 -forms $\}$.

Remark that $b^{+}$in the dimension formula is a topological invariant of $M$ since $b^{+}=1 / 2\left(b_{2}+\tau\right)$, where $b_{2}$ is the second Betti number and $\tau$ is the Hirzebruch index of $M$.

The Pontrjagin number $p_{1}=p_{1}\left(\mathfrak{g}_{P} \otimes \boldsymbol{C}\right)[M]$ is calculated for each simple Lie group as follows; $p_{1}=4 n k, G=S U(n) ; 4(n-2) k, G=\operatorname{Spin}(n) ; 4(n+1) k, G=S p(n)$; $16 k, G=G_{2} ; 36 k, G=F_{2} ; 48 k, G=E_{6} ; 72 k, G=E_{7} ; 120 k, G=E_{8}$, where $k$ is an integer called the index of the bundle $P([1], \S 8)$.

On the moduli space $\mathscr{M}$ of ASD connections a Riemannian metric is defined by a gauge invariant $L_{2}$ inner product. To investigate geometrical properties of this Riemannian metric is an important matter. It seems indeed one of interesting problems to examine geometry of the moduli space from the viewpoint of holonomy group. The examination is available in the special case where the base space $(M, h)$ has as its holonomy group a unitary or symplectic group, that is, $(M, h)$ is Kähler or endowed with a covariantly constant quaternion structure. In fact, the holonomy group of the Riemannian metric on the moduli space is unitary, when $(M, h)$ is a complex Kähler surface. A similar statement holds for the symplectic case. Namely, applying the momentum map method developed in $\S 3$ we deduce Theorem 2.1 which states that the moduli space holonomy is symplectic when the base space holonomy is $S p(1)$.

This holonomy group argument is also valid for the moduli space of EinsteinHermitian connections on a fixed complex vector bundle and further for the moduli space $\mathscr{M}_{\infty}$ of based anti-instantons over the 4 -sphere $S^{4}$ with the standard metric.

Of course, to get the Riemannian curvature tensor of the Riemannian metric on the moduli space is a subject of great interest. The Riemannian curvature tensor is actually expressed in terms of the Green operators of the Laplace 
operators associated to an ASD connection (formula (4.1) in §4). If the base space $M$ carries a covariantly constant quaternion structure (we say such a space hyperkähler 4-manifold), then an identity relating on the curvature holds (Theorem 4.1) and as a consequence of this identity it is shown that the Riemannian metric on the moduli space is Ricci flat. This identity asserts moreover that the quaternionic bisectional curvature vanishes. The notion of quaternionic bisectional curvature appears in $\S 4$ and is regarded as a quaternionic version of the holomorphic bisectional curvature of a Kähler manifold.

The Poincaré bundle and the index bundles present in a geometrical way finite dimensional vector bundles over the moduli space of ASD connections. A principal bundle $\boldsymbol{P}$ with structure group $G$ over the product space $M \times \mathscr{B}_{\imath r}$, or $M \times \mathscr{M}_{g e n}$, is defined by the $\mathcal{G}$-quotient of the product $P \times \mathcal{A}_{\imath r}$, or $P \times \mathcal{A}_{\bar{g} e n}$, where $A_{g e n}^{-}=\{$generic ASD connections on $P\}$. We call this bundle Poincaré bundle. The restriction of $\boldsymbol{P}$ to $\{x\} \times \mathscr{M}_{g e n}$ can be considered as the framed moduli space $\mathscr{M}_{x}$ at $x \in M$.

As the tangent bundle of the moduli space $\mathscr{M}_{g e n}$ is one of typical index bundles, an index bundle is defined in terms of elliptic operators parametrized by connections on $P$. For a given elliptic operator $\mathscr{D} ; \Gamma^{1} \rightarrow \Gamma^{2}$ associated to a connection $\nabla$ on certain vector bundle $V$, coupling this connection $\nabla$ to connections on $P$ we get infinitely many gauge equivariant elliptic operators $\mathscr{D}_{A}, A \in \mathcal{A}$ so that the formal differences $\left(\operatorname{Ker} \mathscr{D}_{A}\right)-\left(\operatorname{Coker} \mathscr{D}_{A}\right)$ well define a (virtual) vector bundle over the orbit space $\mathscr{B}_{\imath r}$, or $\mathscr{M}_{g e n}$. These bundles are associated to the natural fibration $\mathcal{A}_{\imath r} \rightarrow \mathscr{B}_{\imath r}$ with the fibre $\mathcal{G} / z_{G}$, or its restriction over $\mathscr{M}_{g e n}$, which is equipped with a naturally defined connection $\omega$. So, as is explained in $\S 5$, if the base space is endowed with a covariantly constant quaternion structure, these bundles admit in a canonical sense a holomorphic structure together with an Einstein-Hermitian structure of zero trace ${ }_{g}$ Ricci curvature ( $g$ is the Riemannian metric on the moduli space).

Finally we can make additional arguments on the compactness of the moduli space of $S O(3)$-ASD connections over a hyperkähler 4-manifold $M$. Compared to the $S U(2)$-ASD connection case, the moduli space of $S O(3)$-ASD connections has a different aspect. Actually a phenomenon that the moduli space is compact occurs, when the first Pontrjagin number of the $S O(3)$-bundle $P$ is small (Theorem 6.2).

\section{The Riemannian metric on the moduli space.}

Since the Hodge operator depends on the base metric, the moduli space must reflect primarily geometrical properties of the base space $(M, h)$.

We can actually define a natural Riemannian metric on the moduli space as follows. Since $A$ is affine, the tangent space $T_{A} \mathcal{A}_{\imath r}$ is isomorphic to $\Omega^{1}\left(\mathfrak{g}_{P}\right)_{l}$. On this tangent space an inner product is well defined by 


$$
\begin{gathered}
\langle\beta, \gamma\rangle=\int_{M}(-\operatorname{tr} \otimes h)(\beta, \gamma) d v=\int_{M}(-\operatorname{tr})(\beta \Lambda * \gamma) \\
\beta, \gamma \in \Omega^{1}\left(g_{P}\right)_{l} \quad \text { for } \quad G=S U(n) .
\end{gathered}
$$

For general $G$ we must replace - tr by some adjoint invariant inner product. We see easily that this inner product is gauge invariant. Hence the inner product descends to $\mathcal{B}_{2 r}=\mathcal{A}_{2 r} /\left(G / z_{G}\right)$, the orbit space of irreducible connections on $P$ and its restriction on the generic part $\mathscr{M}_{g e n}$ of $\mathscr{M}$ lying smoothly in $\mathscr{B}_{\imath r}$ provides a Riemannian metric there.

When the base space is Kähler, we have the following Kähler structure theorem.

THEOREM ([15], [17]). Let $(M, h)$ be a compact connected Kähler surface and $G$ a compact simple Lie group. Let $\mathscr{M}_{\text {gen }}$ be the generic part of the moduli space $\mathscr{M}$ of $A S D$ connections on $P=P(M, G)$. Then it admits naturally an integrable almost complex structure with respect to which thus defined Riemannian metric becomes Kähler. Its complex dimension is $\operatorname{dim}_{C} \mathscr{M}_{g e n}=1 / 2 p_{1}\left(\mathfrak{g}_{P} \otimes C\right)[M]-$ $\operatorname{dim}_{R} G\left(1-q(M)+p_{g}(M)\right)$ provided that $\mathscr{M}_{g e n}$ is not empty, where $q(M)$ is the irregularity of $M$ and $p_{g}(M)$ is the geometric genus, $\operatorname{dim}_{C} H^{0}\left(M ; \mathcal{O}\left(K_{M}\right)\right)$ for the line bundle $K_{M}$ of holomorphic 2-forms.

Remarks. (i) We call an ASD connection $A$ generic if it is irreducible and its second cohomology $H_{A}^{+}$associated to the Atiyah-Hitchin-Singer complex $0 \rightarrow \Omega^{0}\left(\mathfrak{g}_{P}\right) \rightarrow \Omega^{1}\left(\mathrm{~g}_{P}\right) \rightarrow \Omega^{+}\left(\mathrm{g}_{P}\right) \rightarrow 0$ vanishes.

(ii) Another definition of the Riemannian metric is given by S. Kobayashi ([20]) and investigations of the Riemannian metric over special base spaces (for example the 4-sphere) are proceeded ([6], [12]). A Riemannian metric of another type is also discussed in [25].

(iii) If a Kähler surface $(M, h)$ has positive scalar curvature or the canonical line bundle $K_{M}$ is holomorphically trivial, then $\mathscr{M}_{g e n}$ coincides with $\mathscr{M} \cap \mathscr{B}_{\imath r}$, the moduli space of irreducible ASD connections so that the singularities of $\mathscr{M}$ arise exactly from reducible ASD connections ([14], Remark 2.1).

(iv) The anti-self-duality of connections is equivalent to the stability of holomorphic vector bundles over an algebraic surface. So $\mathscr{M} \cap \mathcal{B}_{\imath r}$ is in oneto-one correspondence with the moduli $\mathscr{N}_{s t}$ of stable vector bundles with corresponding rank and Chern classes.

This theorem says that if the base holonomy is unitary, then the moduli space holonomy is also unitary. Thus, we can pose the problem:

Suppose that $(M, h)$ has holonomy in group $S U(2) \cong S p(1)$. Is the holonomy of the Riemannian metric on the moduli space $M$ of ASD connections symplectic?

This problem can be rewritten as

Suppose that the base space is hyperkähler. Is it true that the moduli space is also hyperkähler? 
Definition. A Riemannian manifold $(N, g)$ is hyperkähler if there exists on $N$ a quaternion structure which is covariantly constant with respect to the Levi-Civita connection.

We can easily observe from this definition that a hyperkähler manifold has a symplectic group as its holonomy group and hence is Ricci flat Kähler and further has a holomorphic symplectic structure so that $K_{N}$ is trivial (see for example [22], IX, Theorem 4.6 and [3]).

Over a compact hyperkähler 4-manifold the dimension of the moduli space of ASD connections is $p_{1}\left(\mathrm{~g}_{P} \otimes C\right)[M]-4 \varepsilon(M) \times \operatorname{dim}_{R} G$ where $\varepsilon$ (torus) $=0$ and $\varepsilon(K 3$ surface $)=1$. So, the dimension is divisible by four. On the other hand we have another circumstantial evidence to this problem;

THEOREM ([26]). Let $M$ be a complex 2-torus or a K3 surface. If $M$ is algebraic, then the moduli sface of stable sheaves has a holomorphic symplectic structure.

We are able to exhibit actually the following affirmative answer.

THEOREM 2.1 ([15]). Let $P=P(M, G)$ be a principal bundle over a compact hyperkähler 4-manifold $M$ with a compact simple Lie group $G$. Then the generic part $\mathscr{M}_{\text {gen }}$ of the moduli space $\mathscr{M}$ of ASD connections on P carries a hyperkähler structure.

Remarks. (i) This theorem holds for an arbitrary compact simple group, for example, a special orthogonal group $S O(n)$.

(ii) Since $K_{M}$ is holomorphically trivial, an ASD connection is generic if and only if it is irreducible. Thus the singular part of the moduli space $\mathscr{M}$ comes from reducible connections.

(iii) Over a compact Kähler manifold $(N, g)$ an Einstein-Hermitian structure is defined on a holomorphic vector bundle $V \rightarrow N$. A Hermitian fibre metric $\psi$ on $V$ is said to be Einstein-Hermitian if its curvature satisfies $\operatorname{tr}_{g} R^{\psi}=\lambda \operatorname{id}_{E}$ for constant $\lambda$ where $\operatorname{tr}_{g}$ means the trace with respect to the Kähler metric $g$. The fibre metric $\phi$ defines in a certain way the principal bundle $P$ with group $U(n), n=\operatorname{rank}(V)$. In terminology of gauge fields we can formulate an EinsteinHermitian connection on $P$ being equivalent to the Einstein-Hermitian structure on the vector bundle $V$. For more details, refer to [15]. It is shown in [15] that the moduli space of irreducible Einstein-Hermitian connections over a compact hyperkähler 4-manifold inherits a hyperkähler structure in a similar way to the anti-self-dual case.

(iv) The framed moduli space $\tilde{M}$ of anti-instantons over the standard 4sphere $S^{4}$ is investigated in [7] with respect to the Penrose twistor approach. On the other hand there is a one-to-one correspondence between $\tilde{\mathscr{M}}$ and the moduli space of based anti-instantons over $S^{4}$ at the north pole $\infty$. Since the ASD equations (1.2) are conformally invariant and $S^{4}=\boldsymbol{R}^{4} \cup\{\infty\}$ is the con- 
formal compactification of the Euclidean 4-space, we obtain the following fact: the moduli space $\mathscr{M}_{\infty}$ of based anti-instantons over $S^{4}$ carries a quaternion structure which is induced naturally from $\boldsymbol{R}^{4}$ and yields a hyperkähler structure on $\mathscr{M}_{\infty}$ (Theorem ([19])). The space $\mathscr{M}_{\infty}$ (or equivalently $\tilde{\mathscr{M}}$ ) has a bundle structure over $\mathscr{M}$ with group $G$. We observe that this fibration is just the restriction of the so-called Poincaré bundle $\boldsymbol{P} \rightarrow S^{4} \times \mathscr{B}_{\imath r}$ to $\{\infty\} \times \mathscr{M}_{\text {gen }}$ (see $\S 5$ for the definition of the Poincaré bundle).

(v) Besides the Euclidean 4-space there are nontrivial examples of complete open hyperkähler 4-manifolds, Eguchi-Hanson metric, Taub-Nut metric, Multicenter Taub-Nut metrics and spaces which are recently discovered by Kronheimer by using the momentum map ([23]). We can argue also over such spaces the moduli space of ASD connections modulo based gauge actions. These moduli spaces carry similarly a hyperkähler structure ([19]).

\section{The momentum map.}

There are two ways for the proof of Theorem 2.1 which is our main theorem. One is the momentum map method due to primarily Marsden-Weinstein ([24]). Another one is the Hodge decomposition method with the Kuranishi map. In [15] we completed a proof of the theorem by using the latter way. While in the latter case due to the calculation, which is not so easy, we can get explicitly the Riemannian curvature tensor by the aid of the Green operators associated to the Laplacians of the Atiyah-Hitchin-Singer complex. Actually, if we denote by $I_{\imath}$ an almost complex structure on $\Omega^{1}\left(\mathfrak{g}_{P}\right)_{l}$ induced from the corresponding almost complex structure on the base space $M$ of the same symbol, $i=1,2,3$, then each tangent space of the moduli space $\mathscr{M}$, being identified with the first cohomology group $H_{A}^{1}$, is invariant under the operation of any $I_{2}$ in such a way that these $I_{2}$ 's define a quaternion structure on $\mathscr{M}$. That this quaternion structure is parallel with respect to the Levi-Civita connection is derived from an argument on the Hodge theory and the Kuranishi map.

We will give here an alternative proof of the theorem using the momentum map.

Each almost complex structure $I_{\imath}$ of $M$ induces naturally an endomorphism on the bundle $\Lambda^{1}$ of 1 -forms and hence an endomorphism on the tensor product $\Lambda^{1} \otimes \mathrm{g}_{P}$ for which we use the same symbol $I_{\imath}$. So, the space $\mathcal{A}_{\imath r}$ of irreducible connections carries almost complex structures $\left\{I_{1}, I_{2}, I_{3}\right\}$, since $T_{A} \mathcal{A}_{\imath r}=\Omega^{1}\left(\mathrm{~g}_{P}\right)_{l}$.

Define skew symmetric bilinear forms $\omega_{i}, \imath=1,2,3$ on $\Omega^{1}\left(\mathrm{~g}_{P}\right)_{l}$ by

$$
\omega_{i}(\alpha, \beta)=\left\langle I_{\imath} \alpha, \beta\right\rangle, \alpha, \beta \in \Omega^{1}\left(g_{P}\right)_{l}
$$

Note that every gauge transformation commutes with $I_{\imath}$ 's and hence is symplectic with respect to each $\omega_{\imath}$.

Let $A$ be an irreducible connection on the principal bundle $P$. The selfdual curvature $F^{+}(A)$ belongs to $\Omega^{+}\left(\mathfrak{g}_{P}\right)=\oplus_{i=1}^{3}\left(\Omega^{0}\left(\mathfrak{g}_{P}\right) \otimes \theta_{\imath}\right)$ (we abbreviate $l$ ). 
This decomposition of $\Omega^{+}\left(\mathrm{g}_{P}\right)$ corresponds to the decomposition (1.1). Denote by $F_{2}=F_{i}(A) \in \Omega^{0}\left(\mathfrak{g}_{P}\right)$ the $\theta_{i}$-component of $F^{+}(A), F^{+}(A)=\sum_{i=1}^{3} F_{i} \otimes \theta_{2}$ here $F_{i}=$ $\left|\theta_{\imath}\right|^{-2} h\left(F^{+}(A), \theta_{\imath}\right)$ and $h ;\left(\Lambda^{2} \otimes \mathrm{g}_{P}\right) \times \Lambda^{2} \rightarrow \mathrm{g}_{P}$ is the bilinear map given by contraction with the base metric $h$.

We would like to define a momentum map $\mu=\left(\mu_{1}, \mu_{2}, \mu_{3}\right) ; \mathcal{A}_{\imath r} \rightarrow \oplus^{3}\left(\Omega^{0}\left(g_{P}\right)\right) *$ by

$$
\begin{gathered}
\mu_{\imath} ; \mathcal{A}_{2 r} \longrightarrow\left(\Omega^{0}\left(\mathfrak{g}_{P}\right)\right)^{*}, \quad \text { the dual space of } \Omega^{0}\left(\mathfrak{g}_{P}\right), \\
\left\langle\mu_{i}(A), \phi\right\rangle=\left|\theta_{\imath}\right|^{2} \int_{M}(-\operatorname{tr})\left(F_{i} \phi\right) d v
\end{gathered}
$$

$\phi \in \Omega^{\circ}\left(\mathrm{g}_{P}\right), i=1,2,3$, where $\langle$,$\rangle in the left hand side denotes the dual pairing.$

Since $\left\langle\mu_{i}(g(A)), \phi\right\rangle=\left\langle\mu_{i}(A), g \cdot \phi \cdot g^{-1}\right\rangle$ for any $g \in \mathcal{G}$, the moduli space $\mathscr{M} \cap \mathscr{B}_{\imath r}$ of irreducible ASD connections on $P$ is then described as $\mathscr{M} \cap \mathscr{B}_{\imath r}=\mu^{-1}(0) /\left(G / z_{G}\right)$, the gauge quotient of the zero locus of the map $\mu$.

THEOREM 3.1. (a) The map $\mu$ is actually a momentum map, namely it satisfies

$$
\left\langle d\left(\mu_{2}\right)_{A}(\beta), \phi\right\rangle=\omega_{i}\left(\beta, \nabla_{A} \phi\right),
$$

$\phi \in \Omega^{0}\left(g_{P}\right), \beta \in \Omega^{1}\left(g_{P}\right)$.

(b) Moreover (i) $\mu$ is $\mathcal{G} / z_{G^{-}}$equivariant,

$$
\mu_{i}(g(A))=a d\left(g^{-1}\right)^{*}\left(\mu_{i}(A)\right), g \in \mathcal{G} / z_{G},
$$

and (ii) the zero set $\mu^{-1}(0)$ is a submanifold of $\mathcal{A}_{2 r}$ and at each $A \in \mu^{-1}(0)$ the tangent space to $\mu^{-1}(0)$ coincides with $\operatorname{Ker}\left(d(\mu)_{A}\right)$ and (iii) the group of gauge transformations $\mathcal{G} / z_{G}$ acts freely on $\mu^{-1}(0)$ and at each $A$ in $\mu^{-1}(0)$ there exists $a$ slice $S_{A} \subset \mu^{-1}(0)$ for this action.

(c) There exist symplectic forms $\left\{\widetilde{\omega}_{1}, \widetilde{\omega}_{2}, \widetilde{\omega}_{3}\right\}$ on the quotient of the zero locus $\mu^{-1}(0) /\left(\mathcal{G} / z_{G}\right)$ satisfying $\pi^{*} \widetilde{\omega}_{i}=j^{*} \omega_{i}, i=1,2,3$, where $j ; \mu^{-1}(0) \rightarrow \mathcal{A}_{\imath r}$ is the canonical embedding and $\pi ; \mu^{-1}(0) \rightarrow \mu^{-1}(0) /\left(G / z_{G}\right)$ is the natural projection.

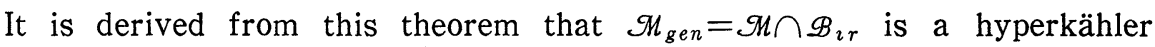
manifold. In fact if we let $\tilde{\nabla}$ be the Levi-Civita connection of the canonical Riemannian metric on $\mathscr{M}_{g e n}$, then each $\widetilde{\omega}_{i}$ is covariantly constant $\left(\tilde{\nabla} \widetilde{\omega}_{i}=0\right)$, since we see that $\tilde{\omega}_{i}(\cdot, \cdot)=\left\langle\tilde{I}_{i}, \cdot,\right\rangle$ holds for the almost complex structure $\tilde{I}_{2}$ on $\mathscr{M} \cap \mathscr{B}_{\imath r}$ induced from $I_{\imath}$ and we have that each $\tilde{I}_{\imath}$ is integrable ([17], §4).

Now we have to show Theorem 3.1. We will first prove (3.3). For any $\beta \in T_{A} \mathcal{A}_{\imath r}\left\langle\left(d \mu_{\imath}\right)_{A}(\beta), \phi\right\rangle$ iseq ual by the definition to $\int_{M}(-\operatorname{tr} \otimes h)\left(d_{A} \beta, \phi \otimes \theta_{i}\right) d v$. The integrand is further calculated as

Hence

$$
\begin{gathered}
(-\operatorname{tr} \otimes h)\left(d_{A} \beta, \phi \otimes \theta_{\imath}\right) d v=(-\operatorname{tr})\left(d_{A} \beta \Lambda *\left(\phi \otimes \theta_{\imath}\right)\right) \\
\quad=d\left(-\operatorname{tr}\left(\beta \Lambda\left(\phi \otimes \theta_{\imath}\right)\right)+(-\operatorname{tr})\left(\beta \Lambda \nabla_{A} \phi \Lambda \theta_{\imath}\right) .\right.
\end{gathered}
$$




$$
\left\langle\left(d \mu_{\imath}\right)_{A}(\beta), \phi\right\rangle=-\int_{M}(-\operatorname{tr} \otimes h)\left(\beta, *\left(\nabla_{A} \phi \Lambda \theta_{\imath}\right)\right) d v .
$$

Using the following formula, which is available by a straightforward calculation, we obtain (3.3)

$$
\text { FORMULA. } \quad *\left(\alpha \Lambda \theta_{\imath}\right)=-I_{i} \alpha, \quad \alpha \in \Lambda^{1} \otimes \mathrm{g}_{P},
$$

The gauge equivariance of $\mu$ is seen from the formula

$$
\left\langle\mu_{i}(g(A)), \phi\right\rangle=\left\langle\mu_{i}(A), g \cdot \phi \cdot g^{-1}\right\rangle .
$$

To prove (ii) and (iii) we can make use of the slice lemma argument ([17], Proposition 2.3) and also the fact that the second cohomology group $H_{A}^{+}$vanishes for each irreducible ASD connection $A$ (see Remark (ii) in $\S 2$ ).

The statement (c) is a direct consequence of [21], Chapter VII, $\S 5$.

\section{Quaternionic bisectional curvature.}

The Riemannian curvature tensor of the canonical Riemannian metric on the moduli space is calculated in [17], $\S 5$.

For tangent vectors $X, Y \in T_{[A]} \mathcal{M}_{g e n}$ the value of the tensor $\langle R(X, Y) Y, X\rangle$ is given by

$$
\begin{aligned}
\langle R(X, Y) Y, X\rangle= & 3\left\langle\{X, Y\}, G_{A}\{X, Y\}\right\rangle-\left\langle[X \Lambda Y]^{+}, G_{A}[X \Lambda Y]^{+}\right\rangle \\
& +\left\langle[X \Lambda X]^{+}, G_{A}[Y \Lambda Y]^{+}\right\rangle
\end{aligned}
$$

Here $\langle\cdot, \cdot\rangle$ denotes the inner product given at (2.1) and $G_{A}$ are the Green operators of the Laplacians $\Delta_{A}$. The bilinear map $\{\cdot, \cdot\}$ is defined by $\{X, Y\}$ $=\sum h^{2 j}\left[X_{\imath}, Y_{j}\right]$ and $[X \Lambda Y]^{+}$denotes the self-dual part of adjoint bundle valued 2-form $[X \Lambda Y]$ for $X=\Sigma X_{i} d x^{2}$ and $Y=\Sigma Y_{i} d x^{2}$.

The above formula applies to any compact Riemannian 4-manifold. But we assume now that the base space $(M, h)$ is hyperkähler. In the hyperkähler case we derive from (4.1) the following

THEOREM 4.1 ([15]). Let $(M, h)$ be a compact hyperkähler 4-manifold and $P$ a principal bundle over $M$ with a compact simple Lie group $G$. Let $\mathscr{M}_{g e n}$ be the moduli stace of generic ASD connections on $P$. Then the curvature tensor of the Riemannian metric on $\mathscr{M}_{g e n}$ satisfies

$$
\langle R(X, Y) Y, X\rangle+\sum_{\imath=1}^{3}\left\langle R\left(X, I_{\imath} Y\right) I_{\imath} Y, X\right\rangle=0,
$$

$X, Y \in T_{[A]} \mathscr{M}_{g e n} \cong H_{A}^{1}$.

This is verified in a direct way by the aid of the following formulae which hold for the hyperkähler case (see [15], §5) 
FormulaE.

$$
[\alpha \Lambda \beta]^{+}+\sum_{\imath=1}^{3}\left[I_{i} \alpha \Lambda I_{i} \beta\right]^{+}=0 \text {, }
$$

and

$$
[\alpha \Lambda \beta]^{+}=\Sigma_{\imath}\left|\theta_{\imath}\right|^{-2}\left\{\alpha, I_{i} \beta\right\} \otimes \theta_{\imath},
$$

$$
G_{A}\left(\phi \otimes \theta_{\imath}\right)=\left|\theta_{\imath}\right|^{-2}\left(G_{A} \phi\right) \otimes \theta_{\imath},
$$

$\alpha, \beta \in \Omega^{1}\left(g_{P}\right), \phi \in \Omega^{0}\left(g_{P}\right)$.

From this we can immediately show that the Ricci curvature identically vanishes, since $\operatorname{Ric}(X, Y)=\Sigma_{j}\left\langle R\left(X, e_{j}\right) e_{j}, Y\right\rangle$ for an orthonormal basis $\left\{e_{j}\right\}$ and we are able to choose

$$
\left\{X_{\imath}, I_{1} X_{\imath}, I_{2} X_{\imath}, I_{3} X_{\imath}\right\}\left(i=1, \cdots, 1 / 4 \operatorname{dim}_{R} M_{g 8 n}\right) \text { as }\left\{e_{j}\right\} .
$$

The formula (4.2) asserts moreover that the left hand side represents a "quaternionic" bisectional curvature of one-dimensional $\boldsymbol{H}$-linear subspaces $V_{X}=$ span $\left\{X, I_{1} X, I_{2} X, I_{3} X\right\}$ and $V_{Y}=\operatorname{span}\left\{Y, I_{1} Y, I_{2} Y, I_{3} Y\right\}$, likewise the holomorphic bisectional curvature of a Kähler manifold ([11]). Actually we can define the quaternionic bisectional curvature of a hyperkähler manifold in the following way

DEFinition. Let $(N, g)$ be a hyperkähler manifold. For any given onedimensional $\boldsymbol{H}$-linear subspaces $V$ and $V^{\prime}$ at $x \in N$ the quaternionic bisectional curvature $Q\left(V, V^{\prime}\right)$ is defined by

$$
Q\left(V, V^{\prime}\right)=g(R(X, Y) Y, X)+\sum_{\imath=1}^{3} g\left(R\left(X, I_{\imath} Y\right) I_{\imath} Y, X\right)
$$

where $X$ and $Y$ are unit vectors in $V$ and $V^{\prime}$, respectively.

It is a simple fact that (4.6) depends only on $\boldsymbol{H}$-invariant subspaces $V, V^{\prime}$.

Theorem 4.1 says then that the quaternionic bisectional curvature of the moduli space vanishes so that this bisectional curvature phenomenon must give a further restriction to the moduli space. We can therefore pose the question:

Does every hyperkähler manifold satisfy this curvature identity?

All hyperkähler 4-manifolds do certainly satisfy it, since they are Ricci flat. With respect to this problem we have a result of $M$. Obata. He obtained in [27], Theorem 3.1 the following fact which says that the Riemannian curvature tensor of a hyperkähler manifold is completely determined in terms of almost complex structures. Let $(N, g)$ be a hyperkähler manifold with a quaternion structure $\{I, J, K=I J\}$. Let $\left(z^{\lambda}\right)$ be a complex coordinate system associated to the first almost complex structure $I$. Then $I, J$ and $g$ take the forms

$$
\begin{aligned}
& I=\left(\begin{array}{cc}
\sqrt{ }-1 \delta_{\lambda}^{\kappa} & 0 \\
0 & -\sqrt{-1} \delta_{\bar{\lambda}}^{\bar{\kappa}}
\end{array}\right) \quad J=\left(\begin{array}{cc}
0 & J_{\hat{\lambda}}^{\bar{\alpha}} \\
J_{\bar{\lambda}}^{\kappa} & 0
\end{array}\right), \\
& J\left(\partial / \partial z^{\lambda}\right)=\sum_{\kappa} J_{\bar{\lambda}}^{\bar{\kappa}} \partial / \partial z^{\bar{\kappa}} \quad \text { and } \quad g=\left(\begin{array}{cc}
0 & g_{\lambda \bar{k}} \\
g_{\bar{\lambda}_{\kappa}} & 0
\end{array}\right) \text {. }
\end{aligned}
$$


The Levi-Civita connection of the metric $g$ whose coefficients are

$$
\Gamma_{\mu \lambda}^{\kappa}=\sum_{\alpha}\left(\partial / \partial z^{\mu} g_{\lambda \bar{\alpha}}\right) g^{\bar{\alpha} \kappa}
$$

coincides with an affine connection $\tilde{\nabla}$ given by

$$
\tilde{\Gamma}_{\mu \lambda}^{\kappa}=-\Sigma_{\alpha}\left(\partial / \partial z^{\mu} J_{\lambda}^{\bar{\alpha}}\right) J_{\bar{\alpha}}^{\kappa}
$$

so that the Riemannian curvature tensor of a hyperkähler manifold can be expressed in terms of the derivatives of the components of $J$ with respect to $\left(z^{\lambda}\right)$. This theorem might give a clue to solve the problem.

\section{Poincaré bundle and index bundles.}

In [18] we investigate the naturally defined connections and their curvature forms on the so-called Poincaré bundle and also on the index bundles.

The Poincaré bundle $\boldsymbol{P}$ is a principal bundle with group $G$ over $M \times \mathcal{B}_{\imath \text { r }}$ defined for a given bundle $P=P(M, G)$ by taking the $\mathcal{G} / z_{G}$-quotient as $\boldsymbol{P}=$ $\left(P \times \mathcal{A}_{\imath r}\right) /\left(\mathcal{G} / z_{G}\right) \rightarrow M \times \mathscr{B}_{\imath r}=G \backslash\left(P \times \mathcal{A}_{\imath r}\right) /\left(\mathcal{G} / z_{G}\right)$, where we regard $\mathcal{G} / z_{G}$ as the group of automorphisms of $P$ covering the identity of $M$ so that it acts on $P \times \mathcal{A}_{\imath r}$ as $(u, A) \mapsto(g(u), g(A))([18])$.

The index bundle is defined over $B_{\imath r}$ as follows (see for the precise definition [18]).

Let $\mathscr{D} ; \Omega^{1}(V) \rightarrow\left(\Omega^{0} \oplus \Omega^{+}\right)(V)$ be a first order elliptic operator associated with a Hermitian vector bundle $V$ over $M$ with an Einstein-Hermitian connection $\nabla$. Tensoring $V$ with an associated vector bundle $\boldsymbol{E}$ to $P$ and also coupling $\nabla$ to $\nabla_{A}, A \in \mathcal{A}_{\imath r}$, we have a family of elliptic operators $\left\{\mathscr{D}_{A} ; \Omega^{1}(V \otimes \boldsymbol{E}) \rightarrow\left(\Omega^{0} \oplus \Omega^{+}\right)\right.$ $(V \otimes \boldsymbol{E})\}$ over the space $\mathcal{A}_{\imath r}$.

We assume that Coker $\mathscr{D}_{A}=0$ for each $A$. Then the dimension of $\operatorname{Ker} \mathscr{D}_{A}$ is constant because of the Atiyah-Singer index theorem and thus we get the family of linear subspaces of the same finite dimension in $\Omega^{1}(V \otimes \boldsymbol{E})$. Since $\mathscr{D}_{A}$ is gauge equivariant, $\left\{\operatorname{Ker} \mathscr{D}_{A}\right\}$ defines a vector bundle over $\mathscr{B}_{\imath r}$ which we call the index bundle.

These bundles are related to an infinite dimensional principal bundle $\mathcal{A}_{\imath r} \rightarrow$ $\mathcal{B}_{\imath r}=\mathcal{A}_{\imath r} /\left(\mathscr{G} / z_{G}\right)$ with structure group $\mathcal{G} / z_{G}$. On this bundle we are able to define in a natural way a connection $\omega$ giving a distribution of horizontal spaces.

In virtue of this connection and its curvature form $\Omega^{\omega}$ we can equip the Poincaré bundle $\boldsymbol{P}$ with a connection $\boldsymbol{A}$ and also any index bundle Ind $\mathscr{D}$ with a connection $\nabla$ ([18]).

Relative to the product structure $M \times \mathcal{B}_{\imath r}$ the curvature $\boldsymbol{F}$ on $\boldsymbol{P}$ decomposes into $\boldsymbol{F}=\boldsymbol{F}^{2,0}+\boldsymbol{F}^{1,1}+\boldsymbol{F}^{0,2}$, where $\boldsymbol{F}^{2,0}=F(A)$, the curvature of $A$ on $P$ at $M \times$ $[A], \boldsymbol{F}^{0,2}=e v_{x}\left(\Omega^{\omega}\right)$ at $\{x\} \times \mathscr{B}_{\imath r}$, evaluation at $x$ of $\Omega^{\omega}$ which takes values in $\Omega^{0}\left(\mathrm{~g}_{P}\right)$, the Lie algebra of $\mathcal{G} / z_{G}$ and $\boldsymbol{F}^{1,1}(Y, \alpha)=-i(Y) \alpha$ for $(Y, \alpha) \in T_{(x,[A])}(M \times$ $\left.\mathscr{B}_{\imath r}\right)\left(Y \in T_{x} M, \alpha\right.$ is a tangent vector to the slice at $A$ ) (see [2] and also [18], §2).

On the other hand on the index bundle Ind $\mathscr{D}$ the curvature $\Omega=\Omega^{\nabla}$ of $\nabla$ is 
written in the form of Gauss equation;

$$
\begin{aligned}
\left(\Omega_{X, Y} \xi, \eta\right)= & \left(\bar{\Omega}_{X, Y} \xi, \eta\right)-\left(\sigma_{X} \xi, \sigma_{Y} \eta\right) \\
& +\left(\sigma_{Y} \xi, \sigma_{X} \eta\right)
\end{aligned}
$$

Here $(\cdot, \cdot)$ is the $L_{2}$-inner product on $\Omega^{1}(V \otimes \boldsymbol{E})$ and $\bar{\Omega}$ is the curvature of the ambient vector bundle $\mathcal{A}_{\imath r} \times \mathcal{G} /{ }_{Z_{G}} \Omega^{1}(V \otimes \boldsymbol{E})$ induced from the principal bundle curvature $\Omega^{\omega}$, and $\sigma_{X} \xi$ denotes the second fundamental form.

We apply these curvature formulae to our hyperkähler 4-manifold case to get the following

THEOREM 5.1. Let $(M, h)$ be a compact hyperkähler 4-manifold and $P$ a principal bundle over $M$ with a compact simple Lie group $G$. Let $\mathscr{M}_{g e n}$ be the moduli space of generic $A S D$ connections on $P$. If we restrict $\boldsymbol{P}$ on $\mathscr{M}_{g e n}$, then the connection $\boldsymbol{A}$ on $\boldsymbol{P}$ yields on any associated complex vector bundle an EinsteinHermitian structure with zero trace ${ }_{g}$-Ricci curvature ( $g$ is the canonical Riemannian metric on $\left.\mathscr{M}_{g e n}\right)$.

Proof. The curvature $\Omega^{\omega}$ of $\omega$ is represented as

$$
\Omega_{X, Y}^{\omega}=-2 G_{A}(\{X, Y\}),
$$

$X, Y \in T_{[\Lambda]} \mathscr{B}_{\imath r}$, where $G_{A}$ denotes the inverse operator of the Laplace operator $\nabla_{A}^{*} \nabla_{A}$ on $\Omega^{0}\left(\mathrm{~g}_{P}\right)([18]$, Proposition 2.1$)$.

We observe that the curvature $\boldsymbol{F}$ on the Poincare bundle $\boldsymbol{P} \rightarrow M \times \mathscr{M}_{\text {gen }}$ and hence on its associated complex vector bundle is of type (1.1) so that it induces a holomorphic structure ([1], Proposition 5.2). To prove that the trace -Ricci $^{-}$ curvature is zero it suffices to show that the trace of $\boldsymbol{F}^{0,2}$ vanishes with respect to the Kähler metric on $\mathscr{M}_{g e n}$, since the $(2,0)$-part $\boldsymbol{F}^{2,0}$ at $[A]$ is just the curvature of $A$ and $A$ is $\mathrm{ASD}$.

LEMMA 5.2. The following equality holds

$$
\left\{X, I_{1} X\right\}+\left\{I_{2} X, I_{3} X\right\}=0, \quad X \in \Omega^{1}\left(g_{P}\right) .
$$

As $\{X, Y\}$ is skew symmetric and $[X \Lambda Y]^{+}$is symmetric, it follows from (4.4) that $\left\{X, I_{\imath} Y\right\}=-\left\{I_{2} X, Y\right\}$ and hence $\{\cdot, \cdot\}$ is $I_{i}$-invariant. Taking the inner product of (4.3) and the covariantly constant 2-form $\theta_{2}$ and using (4.4), we have (5.3).

We will return to the proof of Theorem 5.1. From (5.2) the trace -Ricci curvature is $\operatorname{tr}_{g} F^{0,2}=-2 G_{A}\left(\sum_{j}\left\{Z_{\jmath}, \bar{Z}_{j}\right\}\right)$ for a unitary base $\left\{Z_{j}\right\}$ of $T_{[A]}^{1,0} \mathcal{M}_{g e n}$. Since the real tangent space $T_{[A]} \mathscr{M}_{g e n}$ carries a quaternion structure $\left\{I_{1}, I_{2}, I_{3}\right\}$, we can put $Z_{j}=1 / \sqrt{2}\left(Y_{j}-\sqrt{-1} I_{1} Y_{j}\right), Z_{n+j}=1 / \sqrt{2}\left(I_{2} Y_{j}-\sqrt{-1} I_{1} I_{2} Y_{j}\right), j=1, \cdots$, $n=1 / 4 \operatorname{dim}_{R} T \mathscr{M}_{g e n}$ in such a way that the trace ${ }_{g}$ vanishes from the lemma.

Theorem 5.3. Let $(M, h)$ and $P$ be as in Theorem 5.1. Let $\mathscr{D} ; \Omega^{1}(V) \rightarrow$ 
$\left(\Omega^{0} \oplus \Omega^{+}\right)(V)$ be the elliptic operator associated to an Einstein-Hermatian vector bundle $(V, \nabla)$ and $\mathscr{D}_{A}$ 's elliptic operators coupled to connections $A$ on $P$. We assume that Coker $\mathscr{D}_{A}=0$ for any $A$. Then the connection $\nabla$ of the index bundle Ind $\mathscr{D}=\left\{\operatorname{Ker} \mathscr{D}_{A}\right\}$ restricted on the moduli space $\mathscr{M}_{\text {gen }}$ induces on the complexification of Ind $\mathscr{D}$ the holomorphic structure and also an Einstein-Hermitian structure

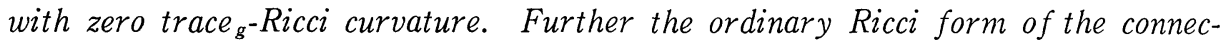
tion $\nabla$, namely of the Einstein-Hermitian structure, is identically zero.

Proof. It is shown ([18], §5) that the curvature $\Omega$ of $\nabla$ is of type $(1,1)$ and hence gives the integrability of a holomorphic vector bundle structure.

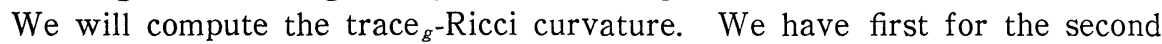
fundamental form $\sigma_{X}$

$$
\left(\sigma_{X} \xi, \sigma_{I_{1} X} \eta\right)+\left(\sigma_{I_{2} X} \xi, \sigma_{I_{3} X} \eta\right)=0,
$$

$\xi, \eta \in \operatorname{Ker} \mathscr{D}_{A}$, because the second fundamental form commutes with each $I_{2}$ (see [18], (5.1) and (5.2) and notice the operation of each $I_{\imath}$ on $\mathrm{g}_{P}$-valued 1-form $X$ ) and also the following holds (see (5.4) in [18]);

$$
\left(\sigma_{X} I_{i} \xi, \sigma_{Y} I_{\imath} \eta\right)=\left(\sigma_{I_{\imath} X} \xi, \sigma_{I_{i} Y} \eta\right)
$$

Then, observing that the curvature $\bar{\Omega}$ of the ambient space is $\Omega^{\omega}$ in a certain sense and applying the Gauss equation (5.1), we see that the trace ${ }_{g}$ must vanish.

To show that the Ricci form $\Phi$ is zero we make use of the Gauss equation again and have

$$
\begin{aligned}
\Phi(Z, \bar{Z})= & \Sigma_{j}\left(\Omega_{Z, \bar{z}} \phi_{\jmath}, \bar{\phi}_{\jmath}\right)=-2 \Sigma_{j}\left(\bar{\Omega}_{X, I_{1} X} \xi_{\jmath}, I_{1} \xi_{\jmath}\right) \\
& +2 \Sigma_{j}\left\{\left(\sigma_{X} I_{1} \xi_{\jmath}, \sigma_{I_{1} X} \xi_{j}\right)-\left(\sigma_{I_{1} X} I_{1} \xi_{\jmath}, \sigma_{X} \xi_{j}\right)\right\}
\end{aligned}
$$

for a unitary basis $\left\{\phi_{i}=1 / \sqrt{2}\left(\xi_{i}-\sqrt{-1} I_{1} \xi_{2}\right)\right\}$ and a tangent vector $Z=X-$ $\sqrt{-1} I_{1} X$ of type (1.0). Like tangent spaces of the moduli space $\mathscr{M}_{g e n}$, each $\operatorname{Ker} \mathscr{D}_{A}$, fibre of Ind $\mathscr{D}$ over $[A]$ enjoys the quaternion structure induced from the $I_{\imath}$ 's. Further each $I_{\imath}$ defined on $\Omega^{1}(V \otimes \boldsymbol{E})$ commutes with the action of infinitesimal gauge transformation $G_{A}(\{X, Y\})$. So, we get

$$
\left(\bar{\Omega}_{X, Y} \xi, I_{1} \eta\right)+\left(\bar{\Omega}_{X, Y} I_{2} \xi, I_{3} \eta\right)=0 .
$$

In a similar way to (5.3) and (5.6) we have by using the fact that $\sigma_{X}$ commutes with each $I_{\imath}$.

$$
\left(\sigma_{X} \xi, \sigma_{Y} I_{1} \xi\right)+\left(\sigma_{X} I_{2} \xi, \sigma_{Y} I_{3} \xi\right)=0 .
$$

Therefore the Ricci form $\Phi$ is identically zero.

Remark. The moduli space $\mathscr{M}_{x}$ of framed ASD connections at $x$ on $P=$ $P(M, G)$ has a bundle structure over $\mathscr{M}$ and of structure group $G$. In this case the bundle $\mathscr{M}_{x} \rightarrow \mathscr{M}_{\text {gen }}$ has moreover a connection whose curvature is of 
type $(1,1)$ and gives an Einstein-Hermitian structure with zero trace -Ricci curvature. In fact, the space $\mathscr{M}_{x}$ is exactly the Poincaré bundle $\boldsymbol{P}$ restricted over $\{x\} \times \mathscr{M}$ and the connection is $\boldsymbol{A}$ restricted over there so that Theorem 5.1 applies.

\section{Remark on the moduli space of $S O(3)$-ASD connections.}

We now suppose that a bundle $P$ has the structure group $S O(3)$ and investigate the moduli space of $S O(3)$-connections on $P$.

From the Atiyah-Singer index theorem the moduli space has the virtual dimension $-2 l-3\left(1-b_{1}+b^{+}\right)\left(l\right.$ is the first Pontrjagin number $\left.p_{1}(P)[M]\right)$.

Unlike $S U(2)$-bundles we have two topological invariants on $S O(3)$-bundle $P$. The second Stiefel-Whitney class $w_{2}(P) \in H^{2}\left(M ; Z_{2}\right)$ and the first Pontrjagin class $p_{1}(P) \in H^{4}(M ; \boldsymbol{Z})$ characterize $S O(3)$-bundles. Two $S O(3)$-bundles $P$ and $P^{\prime}$ are topologically equivalent when these invariants coincide for them. If $w_{2}(P)=0$, then $P$ comes from an $S U(2)$-bundle and $p_{1}(P)=-4 c_{2}(P)$.

We remark that the adjoint bundle $g_{P}$ is equivalent with the oriented 3plane bundle $S=P \times{ }_{\rho} \boldsymbol{R}^{3}$. The bundle $S$ is reducible when it splits into $L \oplus 1$ with an $S O(2)$-bundle $L$ and a trivial real line bundle 1 . In this case $w_{2}(P)=$ $c_{1}(L) \bmod 2$ and $p_{1}(P)=c_{1}(L)^{2}\left(=\left(w_{2}(P)\right)^{2} \bmod 2\right)$.

A connection $A$ on $P$ defines a covariant derivative $\nabla=\nabla_{A}$ on $S$ satisfying $d(\psi, \phi)=(\nabla \phi, \phi)+(\phi, \nabla \Phi)$ and $\nabla\left(\phi_{1} \Lambda \phi_{2} \Lambda \phi_{3}\right)=0$ for any oriented local orthonormal frame $\left\{\phi_{1}, \phi_{2}, \phi_{3}\right\}$ of $S$. A connection $A$ is reducible when it admits a nonzero covariantly constant section $\phi$. So, $S$ splits into $S=\boldsymbol{R} \phi \oplus \boldsymbol{\phi}^{\perp}$.

If a bundle $P$ admits an ASD connection, then the first Pontrjagin number $l$ is nonpositive because of the Chern-Weil theorem $l=-\frac{1}{4 \pi^{2}} \int \operatorname{tr} F \Lambda F$.

While the moduli space of $S U(2)$-ASD connections has ends, we observe in our $S O(3)$ case with a small $l$ a phenomenon that the moduli space must be compact. See [9] and [10] for the standard references on $S O(3)$-bundles and the moduli space of $S O(3)$-connections.

Since the base space is hyperkähler, the quaternion structure $\left\{I_{1}, I_{2}, I_{3}\right\}$ on $M$ acts in a natural way on the tangent spaces of the moduli space of $S O(3)$ connections.

The following theorem gives an obstruction against the existence of $S O(3)$ ASD connections.

Theorem 6.1. Let $M$ be a complex 2-torus or a K3 surface and $P$ be an $S O(3)$-bundle over $M$ with nonzero $w_{2}$. If the first Pontrjagin number $l$ is odd, then there exist no ASD connections on $P$ with respect to an arbitrary hyperkähler base metric.

Proof. Assume that there is an ASD connection on $P$ with respect to some hyperkähler base metric $h$. Then $A$ must be irreducible, otherwise the 
intersection form $I_{M}$ of the space $M$ must odd. Therefore, $A$ is generic from [14], Proposition 2.3 and Remark 2.1. So, the dimension of the moduli space of generic ASD connections is $-2 l-12 \varepsilon(M)$, whereas from Theorem 2.1 it carries a hyperkähler structure and hence its dimension is divisible by four.

Remark. This theorem might suggest that there exist on $M$ no $S O(3)$ bundles with odd $l$.

Let $P$ be an $S O(3)$-bundle with $w_{2}$ and $l$ over a compact Kähler surface.

That $P$ admits ASD connection if and only if there exists a holomorphic line bundle $L$ satisfying the following conditions;

$$
\begin{aligned}
& c_{1}(L)=w_{2} \bmod 2 \text { and } c_{1}(L)^{2}=l \\
& c_{1}(L) \Lambda\left[\omega_{h}\right]=0
\end{aligned}
$$

First two conditions say that the Whitney sum $L \oplus 1$ gives an $S O$ (3)-bundle equivalent to $P$ and (6.2) asserts that $L \oplus 1$ carries a reducible ASD connection.

If (6.2) fails for any holomorphic line bundle $L$ satisfying (6.1), then there exist no reducible ASD connections on $P$ with respect to the base metric $h$.

For an $S O(3)$-bundle with a small $l$ we have the following compactness argument.

THEOREM 6.2. (i) Let $P$ be an $S O(3)$-bundle with $w_{2} \neq 0$ and $l=-2$ over $a$ compact 2-torus $M$. Choose a flat base Kähler metric $h$ in such a way that $c_{1}(L) \Lambda\left[\omega_{h}\right] \neq 0$ for any holomorphic line bundle $L$ with $c_{1}(L)=w_{2} \bmod 2$ and $c_{1}(L)^{2}=$ -2. Let $\mathscr{M}_{g e n}$ be the moduli space of irreducible $h-A S D$ connections on $P$. Then each connected component of $\mathscr{M}_{\text {gen }}$ is a complex flat torus, provided $\mathscr{M}_{\text {gen }} \neq \phi$.

(ii) Let $P$ be an $S O(3)$-bundle with $w_{2}=0$ and $l=-8$ over a K3 surface. Choose a Ricci flat base Kähler metric $h$ so that $c_{1}(L) \Lambda\left[\omega_{h}\right] \neq 0$ for any holomorphic line bundle $L$ with $c_{1}(L)=w_{2} \bmod 2$ and $c_{1}(L)^{2}=-4$ or -8 . Then each of connected component of the moduli space $\mathscr{M}_{\text {gen }}$ of irreducible $h$-ASD connections on $P$ is a compact hyperkähler 4-manifold provided $\mathscr{M}_{\text {gen }}$ is not empty.

We remark on the existence of the base Kähler metrics. It is actually possible to choose them because, since the condition that (6.2) fails is open and dense, we can perturb the metrics $h$ in a suitable way.

Proof. (i) Since the metric $h$ is flat Kähler, the moduli space is endowed with a 4-dimensional hyperkähler structure. We claim that the moduli space is smooth and compact.

Assume that $\left\{\left[A_{\imath}\right]\right\}$ be a sequence in $\mathscr{M}_{\text {gen }}$ which does not converge. As this can not go to any reducible ASD connection because of the base metric condition, it follows that there are finitely many points $\left\{x_{1}, \cdots, x_{k}\right\}$ in $M$ so that over $M^{\prime}=M \backslash\left\{x_{1}, \cdots, x_{k}\right\} \quad(a)$ each $A_{\imath}$ has a gauge transformation $g_{\imath}$ defined over $M^{\prime}$ and $(b) A_{i}^{\prime}=g_{\imath}\left(A_{\imath}\right)$ converges to an $h$-ASD connection $A_{\infty}$ (see the argument in the proof of [9], Theorem 7.3). Since $\int_{M^{\prime}}\left|F\left(A_{\infty}\right)\right|^{2} \leqq \int_{M}\left|F\left(A_{\imath}\right)\right|^{2}$ 
$=8 \pi^{2}$, from the removability theorem due to K. Uhlenbeck $A_{\infty}$ extends smoothly over the whole space $M$ to an ASD connection on an SO(3)-bundle $P_{\infty}$. The bundle $P_{\infty}$ has $w_{2}\left(P_{\infty}\right)=w_{2}(P)$. The first Pontrjagin number $l_{\infty}$ of $P_{\infty}$ is nonpositive and $\left|l_{\infty}\right| \leqq 2$. Moreover $l_{\infty}=-2 \bmod 4$ by a topological argument $([5]$, Theorem 2). So, this is a contradiction. Hence $\mathscr{M}_{g e n}$ is smooth and compact.

We now assert that $\mathscr{M}_{\text {gen }}$ is locally homogeneous and hence each of its connected components becomes a complex 2-torus with a flat Kähler metric.

In fact the identity component $K$ of the isometry group of $(M, h)$ acts on the moduli space $\mathscr{M}_{g e n}$ through the horizontal lifting and this action induces an infinitesimal deformation $i_{X}(F(A))$ of any ASD connection $A$, where $X$ is a Killing vector field $([4], \S 2)$. Since $i_{X} F$ satisfies the gauge fixing equations $\nabla_{A}^{*}\left(i_{X} F\right)=0$, the action is effective. Hence $\mathscr{M}_{g e n}$ is locally homogeneous, because $\operatorname{dim} \mathscr{M}_{\text {gen }}$ coincides with the dimension of $K$.

(ii) Since the Ricci flat Kähler metric $h$ is hyperkähler, the moduli space $\mathscr{M}_{\text {gen }}$ is hyperkähler. We assume that it has a sequence which does not converge within it. So, as in the proof just above we have an $S O(3)$-bundle $P_{\infty}$ with an ASD connection $A_{\infty}$ whose Pontrjagin number $l_{\infty}$ must be $0,-4$ or -8 . The case $l_{\infty}=0$ is eliminated since it then requires that $P_{\infty}$ must be the product bundle, whereas $w_{2}\left(P_{\infty}\right) \neq 0$. If $l_{\infty}$ is -4 , then the connection $A_{\infty}$ must be irreducible, otherwise there is a holomorphic line bundle $L$ satisfying $c_{1}(L) \Lambda\left[\omega_{h}\right]$ $=0$ and also $c_{1}(L)=w_{2} \bmod 2$ and $c_{1}(L)^{2}=-4$ and this does not occur from the base metric condition. Thus $H^{0}$ and $H^{+}$vanishes for $A_{\infty}$ so that obviously $\operatorname{dim} H_{A \infty}^{1} \geqq 0$. However, the Atiyah-Singer index theorem computes the dimension $\operatorname{dim} H^{1}=-\left(\operatorname{dim} H^{0}-\operatorname{dim} H^{1}+\operatorname{dim} H^{+}\right)=-4$. So, every sequence converges and hence $\mathscr{M}_{\text {gen }}$ is compact.

Remark. It is easily seen from the structure of the intersection form $I_{M}$; $H^{2}(M ; \boldsymbol{Z}) \times H^{2}(M ; \boldsymbol{Z}) \rightarrow \boldsymbol{Z}$ that the $S O(3)$-bundles $P$ with the values $w_{2}$ and $l$ appeared in Theorem 6.2 carry reducible ASD connections for some base hyperkähler metric $h_{0}$. We will discuss whether or not those bundles admit an irreducible ASD connection with respect to a Ricci flat Kähler metric.

Let $A_{0}$ be a reducible $h_{0}$-ASD connection on $P$ over a $K 3$ surface. So, $\operatorname{dim} H_{A_{0}}^{0}=1$ and according to (1.1) $H_{A_{0}}^{+}$is written as

$$
H_{A_{0}}^{+}=\left\{\psi_{1} \otimes \theta_{1}+\psi_{2} \otimes \theta_{2}+\phi_{3} \otimes \theta_{3}, \psi_{i} \in H_{A_{0}}^{0}\right\} .
$$

Then the moduli space of ASD connections on $P$ is around $\left[A_{0}\right]$ described as

$$
\left\{\alpha \in H_{A_{0}}^{1} ;|\alpha|<\varepsilon, \Phi(\alpha)=0\right\} / \mathcal{G}_{A_{0}}
$$

where $\Phi ; H_{A_{0}}^{1} \rightarrow H_{A_{0}}^{+}$is a map given by the Kuranishi map and $\mathcal{G}_{A_{0}}$ denotes the isotropy of $A_{0}$, isomorphic to $U(1)$ ([16], Theorem 1). Since $\Phi$ is approximated by the quadratic $\operatorname{map} \Psi ; H_{A_{0}}^{1} \rightarrow H_{A_{0}}^{+}, \alpha \mapsto \operatorname{pr}_{H^{+}}\left([\alpha \Lambda \alpha]^{+}\right)$and the formula (4.3) holds, the primary part of $\left\langle\Phi, \theta_{\imath}\right\rangle, i=1,2,3$ is an indefinite quadratic form so that $\operatorname{Zero}(\Phi)$ has really 5-dimension near the origin. Therefore there must exist an irreducible $h_{0}$-ASD connection $A$ close to $A_{0}$ on $P$ because there are 
at most finitely many reducible ASD connections when the base space is a $K 3$ surface.

Let $h$ be a Ricci flat Kähler metric which satisfies the following; for any Kähler metric on the path $h_{(t)}=(1-t) h_{0}+t h c_{1}(L) \Lambda\left[\omega_{h_{t}}\right] \neq 0$ where $L$ is an arbitrary holomorphic line bundle with $c_{1}(L)=w_{2} \bmod 2$ and $c_{1}(L)^{2}=-8$.

We seek a solution $\alpha \in \Omega^{1}\left(g_{P}\right)$ to the equation

$$
*_{h} F(A+\alpha)+F(A+\alpha)=0
$$

By the well known theorem of Yau there exists a path $h_{t}$ of Ricci flat Kähler metrics from $h_{0}$ to $h$. To solve (6.4) use the continuity method with respect to $h_{t}$;

$$
*_{h_{t}} F(A+\alpha)+F(A+\alpha)=0
$$

We reduce $\left(6.4_{t}\right)$ to

$$
d_{A}^{+, t} \alpha+[\alpha \Lambda \alpha]^{+, t}+1 / 2\left(*_{n_{t}}-*_{h}\right) F(A)=0
$$

where $d_{A}^{+, t} \alpha=1 / 2\left(d_{A} \alpha+*_{h_{t}} d_{A} \alpha\right)$ and $[\alpha \Lambda \alpha]^{+, t}$ is the $*_{h_{t}}$-self-dual part. For $\alpha=\left(d_{A}^{+, t}\right) * \Psi, \Psi \in \Omega^{+, t}\left(g_{P}\right)\left(6.5_{t}\right)$ is written as

$$
d_{A}^{+, t}\left(d_{A}^{+, t}\right) * \Psi+\left[\left(d_{A}^{+, t}\right) * \Psi \Lambda\left(d_{A}^{+, t}\right) * \Psi\right]^{+, t}+1 / 2\left(*_{h_{t}}-*_{h}\right)(F(A))=0
$$

Since $A$ is irreducible and then the operator $d_{A}^{+, t}\left(d_{A}^{+, t}\right)^{*}$ is invertible for small $t$, by applying Taubes' iterating procedure ([13]) we get a solution $\Psi_{t}$ for sufficiently small $t$ in such a way that $A+d_{A}^{+, t * \Psi_{t}}$ is an ASD connection with respect to $h_{t}$. Note that $P$ admit no reducible $h_{t}$-ASD connections because of the Kähler metric condition.

On the other hand there is another way to find an irreducible ASD connection on an $S O(3)$-bundle. In fact for an $S O(3)$-bundle with $l=-6$ over a $K 3$ surface $M$ Donaldson exhibits an irreducible ASD connection on $P$ by pulling back the holomorphic tangent bundle $T P^{2}(\boldsymbol{C})$ by $\pi ; M \rightarrow P^{2}(\boldsymbol{C})$ the double covering $P^{2}(\boldsymbol{C})$ branched over a smooth curve. The moduli space consists only of this single point ([8]).

\section{REFERENCES}

[1] M.F. Atiyah, N. Hitchin and I. M. Singer, Self-duality in four-dimensional Riemannian geometry, Proc. Roy. Soc. London, A 362 (1978) 425-461.

[2] M.F. Atiyah And I.M. Singer, Dirac operators coupled to vector potentials, Proc. Natl. Acad. Sci. U. S. A., 81 (1984) 2597-2600.

[3] A. Beauville, Variétés kähleriennes dont la première classe de Chern est nulle, J. Diff. Geom., 18 (1983) 755-782.

[4] J.P. Bourguignon And H. B. Lawson JR., Stability and isolation phenomena for Yang-Mills fields, Commun. Math. Phys., 79 (1981) 189-230.

[5] A. Dold AND H. Whitney, Classification of oriented sphere bundles over a 4 complex, Ann. Math., 69 (1959) 667-677. 
[6] H. Doi, Y. Matsumoto and T. Matumoto, An explicit formula of the metric on the moduli space of BPST-instantons over $S^{4}$, A Fête of Topology, Academic Press, 1987.

[7] S.K. Donaldson, Instantons and geometric invariant theory, Commun. Math. Phys., 93 (1984) 453-460.

[8] S.K. Donaldson, Polynomial invariants for smooth four-manifolds, preprint.

[9] R. Fintushel and R. Stern, Pseudofree orbifolds, Ann. Math., 122 (1985) 335364.

[10] D. Freed and K. Uhlenbeck, Instantons and four-manifolds, Springer-Verlag, New York, 1984.

[11] S. Goldberg and S. Kobayashi, On holomorphic bisectional curvature, J. Diff. Geom., 1 (1967) 225-233.

[12] D. Groisser and T.H. Parker, The Riemannian geometry of the Yang-Mills moduli space, Commun. Math. Phys., 112 (1987) 663-689.

[13] M. IToH, Self-dual Yang-Mills equations and Taubes' theorem, Tsukuba J. Math., 8 (1984) 1-29.

[14] M. Iтон, The moduli space of Yang-Mills connections over a Kähler surface is a complex manifold, Osaka J. Math., 22 (1985) 845-862.

[15] M. Iтон, Quaternion structure on the moduli space of Yang-Mills connections, Math. Ann., 276 (1987) 581-593.

[16] M. IToH, Singularities of the moduli space of Yang-Mills fields, Proc. Japan Acad., 63 (1987) 170-173.

[17] M. Iтон, Geometry of anti-self-dual connections and Kuranishi map, J. Math. Soc. Japan, 40 (1988) 9-32.

[18] M. ITOH, Yang-Mills connections and the index bundles, to appear in Tsukuba Math. J.

[19] M. Iтон, Based ant1-instantons and gravitational instantons, in preparation.

[20] S. Kobayashi, Submersions of $C R$ submanifolds, Tôhoku Math. J., 39 (1987).

[21] S. Kobayashi, Differential geometry of complex vector bundles, Iwanami, 1987.

[22] S. Kobayashi and K. Nomizu, Foundations of differential geometry, II, Interscience, 1969.

[23] P.B. Kronheimer, The construction of gravitational instantons as hyperkähler quotients, preprint.

[24] J. Marsden And A. D. Weinstein, Reduction of symplectic manifolds with symmetry, Reports on Math. Phys., 5 (1974) 121-130.

[25] T. Matumoto, On the metric of type II on the moduli space, 1985.

[26] S. MukaI, Symplectic structure of the moduli space of sheaves on an abelian or K3 surface, Invent. math., 77 (1984) 101-116.

[27] M. OватA, Hermitian manifolds with quaternıon structure, Tôhoku Math. J., 10 (1958) 11-18.

InSTITUTE of Mathematics

UNIVERSITY OF TSUKUBA

305, Tsukuba, Japan 